\title{
Integral-Equation Analysis of 3-D Metallic Objects Arranged in 2-D Lattices Using the Ewald Transformation
}

\author{
Ivica Stevanović, Member, IEEE, Pedro Crespo-Valero, Katarina Blagović, Frédéric Bongard, and \\ Juan R. Mosig, Fellow, IEEE
}

\begin{abstract}
We present a space-domain integral-equation method for the analysis of periodic structures formed by three-dimensional (3-D) metallic objects arranged in a general skewed two-dimensional lattice. The computation of the space-domain Green's function is accelerated using the Ewald transformation. The method is validated on several periodic structures ranging from planar frequency-selective surfaces to 3-D photonic crystals and metamaterials. For these structures, our technique shows a clear advantage in terms of computational speed when compared with available commercial softwares.
\end{abstract}

Index Terms-Frequency-selective surfaces (FSSs), Green's functions (GFs), integral equations (IEs), metamaterials, periodic structures, photonic-bandgap (PBG) materials.

\section{INTRODUCTION}

$\mathbf{P}$ ERIODIC structures, such as frequency-selective surfaces (FSSs) and photonic-bandgap (PBG) materials, provide uninhibited transmission in specific frequency bands while suppressing transmission in other bands. They have found a variety of applications in a broad range of the electromagnetic (EM) spectrum including antenna reflectors, quasi-optical filters, polarizers, switches, and designing more efficient lasers [1]. Recently, it has been shown that periodic structures built from nonmagnetic conducting sheets can exhibit negative real parts of permittivity and permeability [2]. These materials, designated as left-handed metamaterials, open new application fields.

The scattering from doubly periodic arrays of perfect electric conductor (PEC) objects has been simulated using source-model [4] or time-domain [5] techniques. Fully three-dimensional (3-D) modeling capabilities of periodic structures can be achieved using finite-element (FE) methods that can cope with complicated shapes and anisotropic materials. However, the radiation into unbounded regions asks for either absorbing boundary conditions (ABCs) or perfectly matched layers (PMLs) and special measures need to be taken into account when employing these conditions to the scattered field formulation [6]. Therefore, hybrid finite-element/boundary-integral $(\mathrm{FE} / \mathrm{BI})$ techniques that are based on $\mathrm{FE}$ modeling of

Manuscript received February 1, 2006; revised May 30, 2006. This work was supported in part by the European Union Network of Excellence Metamorphose under Grant NMTP3-CT-2004-500252.

The authors are with the Laboratory of Electromagnetics and Acoustics, Ecole Polytechnique Fédérale de Lausanne, CH-1015 Lausanne, Switzerland (e-mail: ivica.stevanovic@epfl.ch; juan.mosig@epfl.ch).

Digital Object Identifier 10.1109/TMTT.2006.882876 a unit cell and BI modeling of its top and bottom faces are introduced. In these methods, the BI fields are expanded using spectral-domain Floquet modes [7] or using space-domain Green's functions (GFs) accelerated applying the Ewald transformation [8].

Periodic structures are advantageously analyzed with integral-equation (IE) techniques, usually formulated in the spectral domain, as exemplified by the case of multilayered FSSs [1]. Two approaches can be employed. One is to compute a specific FSS in its entirety [9] and the other is to cascade the generalized scattering matrices (GSMs) of the constitutive building blocks [10]. Recently, the boundary-integral-resonant-mode expansion (BI-RME), used to compute the entire-domain basis functions in conjunction with method of moments (MoM), has been applied to analysis of FSSs [11].

The scope of these IE techniques is limited to planar metallic scatterers embedded in the planes of periodicity. Moreover, the involved EM quantities are expressed as Floquet sums with a slow convergence rate. In this paper, we propose a spatial-domain formulation of the IE, which allows us to deal with arbitrary 3-D metallic objects distributed on a two-dimensional (2-D) generic nonorthogonal lattice. Furthermore, the involved potential GFs are computed using the Ewald transformation to accelerate their convergence.

Among the different techniques to speed up the evaluation of GFs [12], the Ewald transformation has clearly demonstrated its suitability for periodic problems, efficiently combining the spectral and spatial formulations of the GFs. It has been successfully used in the efficient GF computation of 2-D free-space periodic structures with rectangular lattices [13]-[15] and multilayered planar periodic structures [16], where the approach involves the approximation of spectral-domain GFs by the generalized pencil-of-function (GPOF) method [17]. In [18], a new acceleration technique with an exponential convergence rate has been presented, which is easier to implement numerically and can perform better than Ewald transformation for moderate accuracies. However, the Ewald transformation remains better for higher accuracy, the only inconvenience being in the computation of the complementary error functions with complex arguments. Our approach obviates this computational complexity by storing the GFs into 3-D tables. The GF values needed to compute the numerical integrals are retrieved from the tables using efficient interpolation routines, which leads to very accurate results and much faster computation than if the GFs are evaluated directly. 


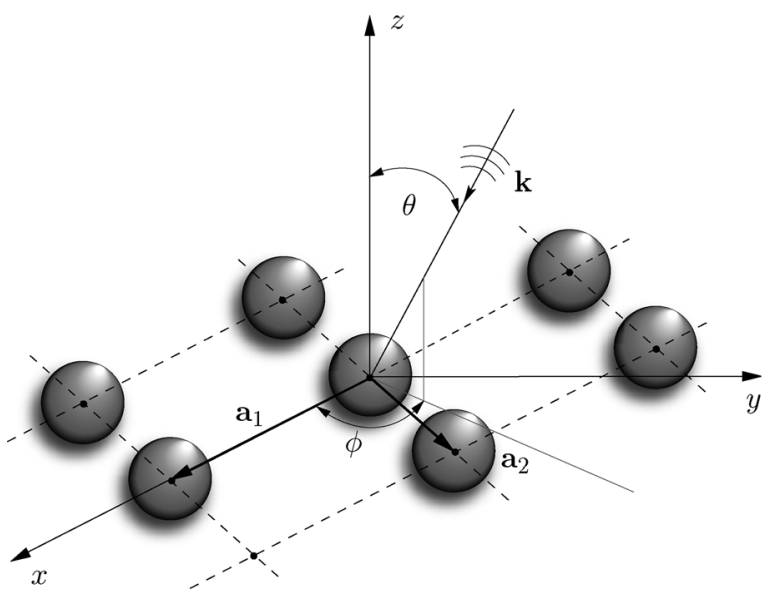

Fig. 1. 2-D periodic structure with a general skewed lattice.

We apply the Ewald transformation to 3-D geometries periodically repeated along a 2-D skewed lattice. However, this technique can be easily extended to the case of 3-D metallo-dielectric inclusions in a 2-D lattice either by using the equivalence theorem and electric and magnetic surface currents or by means of the volume IEs and polarization currents. Both approaches have the convenience of dealing with homogeneous GFs where the Ewald transformation can be easily applied.

\section{IE AND MoM}

We consider a periodic structure with identical 3-D metallic objects of arbitrary shape periodically repeated in the $x y$-plane (Fig. 1). The periodic structure has a general skewed lattice defined by primitive vectors $\mathbf{a}_{1}$ and $\mathbf{a}_{2}$ and is illuminated by a plane wave impinging with the incidence angles $(\theta, \phi)$. Let $S$ designate the surface of the perfectly conducting 3-D object within the structure's unit cell. The analysis is based on the solution of the following mixed-potential integral equation (MPIE) [19]:

$$
-\hat{n} \times \mathbf{E}^{\mathrm{i}}=\hat{n} \times\left(-\mathrm{j} \omega \overleftrightarrow{\mathbf{G}}_{A} \otimes \mathbf{J}+\frac{1}{\mathrm{j} \omega}\left(\nabla G_{V}\right) \otimes\left(\nabla^{\prime} \cdot \mathbf{J}\right)\right)
$$

obtained by enforcing the boundary conditions (BCs) on the PEC surface $S$. In (1), $\mathbf{E}^{\mathrm{i}}$ is the incident electric field, $\hat{n}$ is the outside normal to $S, \mathbf{J}$ is the unknown current density on $S$, and $\overleftrightarrow{\mathbf{G}}_{A}$ and $G_{V}$ are the vector and scalar potential GFs that take into account the periodicity of the problem. Finally, $\otimes$ is a shorthand notation [20] of the surface convolution integral

$$
\overleftrightarrow{\mathbf{G}} \otimes \mathbf{J}=\int_{S^{\prime}} \overleftrightarrow{\mathbf{G}}\left(\mathbf{r} \mid \mathbf{r}^{\prime}\right) \cdot \mathbf{J}\left(\mathbf{r}^{\prime}\right) \mathrm{d} S^{\prime}
$$

where primed and unprimed coordinates designate source and observer positions, respectively.

Equation (1) is solved by applying the MoM in the Galerkin form. The unknown current density $\mathbf{J}$ is expanded using a set of $N$ Rao-Wilton-Glisson (RWG) [21] basis functions $\mathbf{f}_{k}$ defined on triangular subdomains (or facets) $S_{k}$ of the surface $S$

$$
\mathbf{J}=\sum_{k=1}^{N} \alpha_{E k} \mathbf{f}_{k}
$$

where $\alpha_{E k}$ are the unknown coefficients. By substituting (2) into (1) and testing the IEs by the same set of basis functions $\mathbf{f}_{k}$, for each frequency $\omega$, the following system of linear equations is obtained:

$$
\sum_{k=1}^{N}\left(a_{i, k}+v_{i, k}\right) \alpha_{E k}=e_{k}
$$

where for $i, k=1, \ldots, N$

$$
\begin{aligned}
e_{k} & =\int_{S} \mathbf{E}^{\mathrm{i}}(\mathbf{r}) \cdot \mathbf{f}_{k}(\mathbf{r}) \mathrm{d} S=\left\langle\mathbf{E}^{\mathrm{i}}, \mathbf{f}_{k}\right\rangle \\
a_{i, k} & =\mathrm{j} \omega\left\langle\mathbf{f}_{i}, \overleftrightarrow{\mathbf{G}}_{A} \otimes \mathbf{f}_{k}\right\rangle, v_{i, k}=\frac{1}{\mathrm{j} \omega}\left\langle\nabla \cdot \mathbf{f}_{i}, G_{V} \otimes\left(\nabla^{\prime} \cdot \mathbf{f}_{k}\right)\right\rangle .
\end{aligned}
$$

The MPIEs involve potential GFs that have milder singularity ( $R^{-1}$ instead of $R^{-3}$ for field GFs), which allows for the above integrals to be numerically solved in two possible ways. The singularity can be extracted, computed analytically [22], [23], and added to the remaining regular part. Another strategy is to solve the integral in polar coordinates, where the Jacobian of the transformation will cancel out the singularity [20].

\section{REFLECTION AND TRANSMISSION COEFFICIENTS OF A PERIODIC STRUCTURE}

The components of the total EM field transverse to the propagation direction can be expanded in any plane $z$ of the periodic structure using the transverse vector eigenfunctions, designated as Floquet modes $\left(\mathbf{e}_{\mathrm{t} q}, \mathbf{h}_{\mathrm{t} q}\right)$ as [24], [25]

$$
\begin{aligned}
& \mathbf{E}=\sum_{q}\left(a_{q} \mathrm{e}^{-\gamma_{q} z}+b_{q} \mathrm{e}^{\gamma_{q} z}\right) \sqrt{Z_{q}} \mathbf{e}_{\mathrm{t} q} \\
& \mathbf{H}=\sum_{q}\left(a_{q} \mathrm{e}^{-\gamma_{q} z}-b_{q} \mathrm{e}^{\gamma_{q} z}\right) / \sqrt{Z_{q}} \mathbf{h}_{\mathrm{t} q}
\end{aligned}
$$

where $q$ goes through all the $\mathrm{TE}_{m, n}$ and $\mathrm{TM}_{m, n}$ modes, $a_{q}$ and $b_{q}$ are the power coefficients of the modes propagating in the positive and negative $z$-directions, respectively, $\gamma_{q}$ is the propagation constant, and $Z_{q}$ is the characteristic impedance of the $q$ th mode given by

$$
Z_{q}= \begin{cases}\frac{\mathrm{j} \omega \mu}{\gamma_{q}}, & \text { for TE mode } \\ \frac{\gamma_{q}}{\mathrm{j} \omega \varepsilon}, & \text { for TM mode. }\end{cases}
$$

In the case of oblique incidence $(\theta \neq 0)$, an arbitrary polarized incident plane wave can be expressed as a combination of the two fundamental Floquet modes, namely, $\mathrm{TE}_{00}$ and $\mathrm{TM}_{00}$, while in the case of normal incidence $(\theta=0)$, the incident plane wave is the Floquet TEM mode [26]. The expressions for the transversal and longitudinal components of the Floquet modes of a general skewed lattice are compiled in Appendix A.

Suppose that the incident plane wave impinging from below corresponds to the $p$ th Floquet mode, the transverse component of the electric field of this wave can then be expressed as

$$
\mathbf{E}^{\mathrm{i}}=a_{p}^{\mathrm{i}} \mathrm{e}^{-\gamma_{p} z} \sqrt{Z_{p}} \mathbf{e}_{\mathrm{t} p} .
$$

Let $S_{\mathrm{r}}$ be a unit surface in the $z=z_{\mathrm{r}}$ plane for which the reflection coefficient is being computed, and let $S_{\mathrm{t}}$ be the surface in 
the $z=z_{\mathrm{t}} \geq z_{\mathrm{r}}$ plane for which the transmission coefficient is sought for. The total electric field in $S_{\mathrm{r}}$ consists of the incident field traveling in the positive $z$-direction and the field scattered from the metallic objects traveling in the negative $z$-direction, and its transverse component can be expressed as

$$
\mathbf{E}\left(z=z_{\mathrm{r}}\right)=a_{p}^{\mathrm{i}} \mathrm{e}^{-\gamma_{p} z_{\mathrm{r}}} \sqrt{Z_{p}} \mathbf{e}_{\mathrm{t} p}+\mathbf{E}^{\mathrm{r}}
$$

This field can be expanded into Floquet modes using (4a)

$$
\mathbf{E}\left(z=z_{\mathrm{r}}\right)=\sum_{q}\left(a_{q} \mathrm{e}^{-\gamma_{q} z_{\mathrm{r}}}+b_{q} \mathrm{e}^{\gamma_{q} z_{\mathrm{r}}}\right) \sqrt{Z_{q}} \mathbf{e}_{\mathrm{t} q} .
$$

Multiplying both (6) and (7) by $\mathbf{e}_{\mathrm{t} p}^{*}$, integrating over the surface of the unit cell $S_{\mathrm{r}}$, and using the orthonormal property of the Floquet modes $\int_{S_{\mathrm{r}}} \mathbf{e}_{\mathrm{t} q} \cdot \mathbf{e}_{\mathrm{t} p}^{*} \mathrm{~d} S=\delta_{p q}$, the reflection coefficient of the $p$ th mode can be expressed in terms of the reflected field as follows:

$$
\rho=\frac{b_{p}}{a_{p}^{\mathrm{i}}}=\frac{\mathrm{e}^{-\gamma_{p} z_{\mathrm{r}}}}{a_{p}^{\mathrm{i}} \sqrt{Z_{p}}} \int_{S_{\mathrm{r}}} \mathbf{E}^{\mathrm{r}} \cdot \mathbf{e}_{\mathrm{t} p}^{*} \mathrm{~d} S .
$$

In the same manner, we obtain the transmission coefficient. The total electric field in $S_{\mathrm{t}}$ consists of the incident electric field and the scattered field $\mathbf{E}^{\mathrm{t}}$ both propagating in the positive $z$-direction

$$
\mathbf{E}\left(z=z_{\mathrm{t}}\right)=a_{p}^{\mathrm{i}} \mathrm{e}^{-\gamma_{p} z_{\mathrm{t}}} \sqrt{Z_{p}} \mathbf{e}_{\mathrm{t} p}+\mathbf{E}^{\mathrm{t}}
$$

In $z>z_{\mathrm{t}}$, there are only waves propagating in the positive $z$-direction so the expansion of the total electric field can be expressed as

$$
\mathbf{E}\left(z=z_{\mathrm{t}}\right)=\sum_{q} a_{q} \mathrm{e}^{-\gamma_{q} z_{\mathrm{t}}} \sqrt{Z_{q}} \mathbf{e}_{\mathrm{t} q} .
$$

Multiplying (9) and (10) by $\mathbf{e}_{\mathrm{t} p}^{*}$, integrating over $S_{\mathrm{t}}$, and taking into account the orthonormality of the Floquet modes, for the transmission coefficient, one obtains

$$
\tau=\frac{a_{p}}{a_{p}^{\mathrm{i}}}=1+\frac{\mathrm{e}^{\gamma_{p} z_{\mathrm{t}}}}{a_{p}^{\mathrm{i}} \sqrt{Z_{p}}} \int_{S_{\mathrm{r}}} \mathbf{E}^{\mathrm{t}} \cdot \mathbf{e}_{\mathrm{t} p}^{*} \mathrm{~d} S .
$$

The transverse component of the scattered electric field can be expressed as a convolution integral of induced electric currents and the corresponding GF as

$$
\mathbf{E}=\int_{S^{\prime}} \overleftrightarrow{\mathbf{G}}_{E E}\left(\mathbf{r} \mid \mathbf{r}^{\prime}\right) \cdot \mathbf{J}\left(\mathbf{r}^{\prime}\right) \mathrm{d} S^{\prime}
$$

The dyadic $\overleftrightarrow{\mathbf{G}}_{E E}$ relates the electric current density to the transverse electric field and is given by

$$
\overleftrightarrow{\mathbf{G}}_{E E}\left(\mathbf{r} \mid \mathbf{r}^{\prime}\right)=\sum_{q} \widetilde{V}_{q}\left(z, z^{\prime}\right) \mathbf{e}_{\mathrm{t} q}(x, y) \cdot \mathbf{e}_{q}^{*}\left(x^{\prime}, y^{\prime}\right)
$$

where $\mathbf{e}_{q}=\mathbf{e}_{t q}+\mathbf{e}_{z q}$. The spectral domain quantity $\widetilde{V}_{q}$ represents the voltage at the point $z$ along the equivalent transmission line for a unitary value of the current generator placed at $z=z^{\prime}$

$$
\widetilde{V}_{q}\left(z, z^{\prime}\right)=\frac{Z_{q}}{2} \mathrm{e}^{-\gamma_{q}\left|z-z^{\prime}\right|}
$$

Finally, once the unknowns $\alpha_{E k}$ have been computed by solving the MoM system of (3), using the orthonormality of the Floquet modes and (2) and (12a)-(12c), the reflection and transmission coefficients of any Floquet mode can be found as

$$
\begin{aligned}
\rho= & \frac{\sqrt{Z_{p}} \mathrm{e}^{-\gamma_{p} z_{\mathrm{r}}}}{2 a_{p}^{\mathrm{i}}} \sum_{k} \alpha_{E k} \\
& \times \int_{S_{k}} \mathrm{e}^{-\gamma_{p} \mid z_{\mathrm{r}}-z^{\prime}} \mathbf{f}_{k}\left(x^{\prime}, y^{\prime}, z^{\prime}\right) \cdot \mathbf{e}_{p}^{*}\left(x^{\prime}, y^{\prime}\right) \mathrm{d} S^{\prime} \\
\tau= & 1+\frac{\sqrt{Z_{p}} \mathrm{e}^{\gamma_{p} z_{\mathrm{t}}}}{2 a_{p}^{\mathrm{i}}} \sum_{k} \alpha_{E k} \\
& \times \int_{S_{k}} \mathrm{e}^{-\gamma_{p} \mid z_{\mathrm{t}}-z^{\prime}} \mathbf{f}_{k}\left(x^{\prime}, y^{\prime}, z^{\prime}\right) \cdot \mathbf{e}_{p}^{*}\left(x^{\prime}, y^{\prime}\right) \mathrm{d} S^{\prime} .
\end{aligned}
$$

In the case of a periodic structure with identical planar apertures of arbitrary shape periodically perforated in the $z=0$ plane, the reflection and transmission coefficients are computed as shown in [27].

\section{2-D PERIODIC GFs}

\section{A. Image Formulation}

Suppose the periodic structure is illuminated by a plane wave impinging from below with the incident angles $\theta, \phi$. The propagation vector of this plane wave is defined as

$$
\mathbf{k}=k \sin \theta \cos \phi \hat{x}+k \sin \theta \sin \phi \hat{y}+k \cos \theta \hat{z}=\mathbf{k}_{\rho}+\mathbf{k}_{z} .
$$

All the cells in the periodic structure are equal, and the field impinging on them is the same, save for a phase shift. This means that the total EM field (i.e., the solution of the Maxwell's equations) will be equal for all the cells, except for the phase shift that corresponds to that one of the excitation. Therefore, if any EM-field component is known on the original cell and given by $f_{0}(\mathbf{r})$, it will be known over the entire periodicity plane since it can be written as

$$
f(\mathbf{r})=\sum_{m, n=-\infty}^{\infty} f_{0}\left(\mathbf{r}-\boldsymbol{\rho}_{m n}\right) \mathrm{e}^{-\mathbf{j} \mathbf{k} \cdot \boldsymbol{\rho}_{m n}}
$$

where the exponent reflects the phase shifts imposed by the excitation and $\boldsymbol{\rho}_{m n}=m \mathbf{a}_{1}+n \mathbf{a}_{2}$ is the translation vector of the lattice.

The same considerations apply to GFs. Function $f_{0}$ can be a field component, but also any EM quantity linearly related to the fields (potentials, surface currents, etc.) Therefore, if $G_{0}$ 
represents the free-space GF or the GF without periodic BCs, the GF of a periodic structure can be written as

$$
G(\mathbf{R})=\sum_{m, n} G_{0}\left(\mathbf{R}_{m n}\right) \mathrm{e}^{-\mathrm{j} \mathbf{k} \cdot \boldsymbol{\rho}_{m n}}
$$

where $\mathbf{R}=\mathbf{r}-\mathbf{r}^{\prime}$ and $\mathbf{R}_{m n}=\mathbf{R}-\boldsymbol{\rho}_{m n}$.

\section{B. Modal Formulation}

Let $\mathbf{k}_{1}$ and $\mathbf{k}_{2}$ be the vectors of the reciprocal lattice defined by

$$
\mathbf{k}_{1}=2 \pi \frac{\mathbf{a}_{2} \times\left(\mathbf{a}_{1} \times \mathbf{a}_{2}\right)}{\left|\mathbf{a}_{1} \times \mathbf{a}_{2}\right|^{2}} \quad \mathbf{k}_{2}=2 \pi \frac{\mathbf{a}_{1} \times\left(\mathbf{a}_{2} \times \mathbf{a}_{1}\right)}{\left|\mathbf{a}_{1} \times \mathbf{a}_{2}\right|^{2}} .
$$

Applying the Poisson 2-D summation formula to (14), one obtains the modal formulation of the periodic GF

$$
G(\mathbf{R})=\frac{1}{\Omega} \sum_{m, n} \widetilde{G}_{0}\left(\mathbf{K}_{m n}, z-z^{\prime}\right) \mathrm{e}^{\mathrm{j} \mathbf{K}_{m n} \cdot \mathbf{R}}
$$

where $\Omega=\left|\mathbf{a}_{1} \times \mathbf{a}_{2}\right|$ represents the surface of the unit cell, $\mathbf{k}_{m n}=m \mathbf{k}_{1}+n \mathbf{k}_{2}$ is the translation vector of the reciprocal lattice, $\mathbf{K}_{m n}=\mathbf{k}_{m n}-\mathbf{k}_{\rho}$, and $\widetilde{G}_{0}\left(k_{\rho}, z-z^{\prime}\right)=\mathcal{F}\left\{G_{0}\left(\rho, z-z^{\prime}\right)\right\}$. If the periodic structure is in free space, we have the following Fourier transformation pair:

$$
G_{0}=\frac{1}{4 \pi} \frac{\mathrm{e}^{-\mathrm{j} k R}}{R} \quad \rightleftarrows \quad \widetilde{G}_{0}=\frac{\mathrm{e}^{-\gamma\left|z-z^{\prime}\right|}}{2 \gamma}
$$

and the required GFs are $G_{V 0}=G_{0} / \varepsilon_{0}$ and $\overleftrightarrow{\mathbf{G}}_{A 0}=\mu_{0} G_{0} \overleftrightarrow{\mathbf{I}}$ for electric scalar and magnetic vector potentials, and $G_{W 0}=G_{0} / \mu_{0}$ and $\overleftrightarrow{\mathbf{G}}_{F 0}=\varepsilon_{0} G_{0} \overleftrightarrow{\mathbf{I}}$ for magnetic scalar and electric vector potentials, respectively. In (17), $R=|\mathbf{R}|$, $\gamma=\sqrt{k_{x}^{2}+k_{y}^{2}-k^{2}}$, and $\overleftrightarrow{\mathbf{I}}=\hat{x} \hat{x}+\hat{y} \hat{y}+\hat{z} \hat{z}$.

\section{Potential GFs AND Ewald TRANSFORMation}

Both infinite sums in (14) and (16) are slowly convergent. The image sum is converging faster when the observer point is in the source point region since the singularity is perfectly included in the expression. However, close to the lateral periodic walls, the convergence is deteriorated as the periodic BCs are going to be satisfied only for an infinite number of images taken into account. Each term of the modal sum, on the other hand, satisfies the BCs at the lateral walls and, hence, the convergence of this sum is faster close to the walls. However, taking into account the singularity at the source point would require an infinite number of modes leading, therefore, to the slow convergence of the modal sum in the source point region.

The Ewald method successfully combines both modal and image formulations into a fast converging series. Here, we present the details of the Ewald transform used to compute the potential GFs of a periodic structure with general skewed lattice. According to (14) and (17), a potential GF of such a structure is given by the following image sum:

$$
G(\mathbf{R})=\frac{1}{4 \pi} \sum_{m, n} \frac{\mathrm{e}^{-\mathrm{j} k R_{m n}}}{R_{m n}} \mathrm{e}^{-\mathrm{j} \mathbf{k} \cdot \boldsymbol{\rho}_{m n}} .
$$

Following the results of Ewald [13], [28], we use the identity

$$
\frac{\mathrm{e}^{-\mathrm{j} k R}}{R}=\frac{2}{\sqrt{\pi}} \int_{0}^{\infty} \mathrm{e}^{-R^{2} \xi^{2}+\frac{k^{2}}{4 \xi^{2}}} \mathrm{~d} \xi
$$

where $\xi$ is a complex variable and the path of integration is chosen such that the integrand remains bounded as $\xi \rightarrow 0$ and decays as $\xi \rightarrow \infty$. Splitting the integration path at $E$ in (19), we can write

$$
\Psi=\Psi_{1}+\Psi_{2}
$$

with

$$
\Psi_{1}=\frac{1}{4 \pi} \sum_{m, n} \mathrm{e}^{-\mathbf{j k} \cdot \boldsymbol{\rho}_{m n}} \frac{2}{\sqrt{\pi}} \int_{0}^{E} \mathrm{e}^{-R_{m n}^{2} \xi^{2}+\frac{k^{2}}{4 \xi^{2}}} \mathrm{~d} \xi
$$

and

$$
\Psi_{2}=\frac{1}{4 \pi} \sum_{m, n} \mathrm{e}^{-\mathrm{j} \mathbf{k} \cdot \boldsymbol{\rho}_{m n}} \frac{2}{\sqrt{\pi}} \int_{E}^{\infty} \mathrm{e}^{-R_{m n}^{2} \xi^{2}+\frac{k^{2}}{4 \xi^{2}}} \mathrm{~d} \xi .
$$

Using the Poisson 2-D summation formula and the following identity [29]:

$$
\begin{aligned}
& \int_{r}^{\infty} \mathrm{e}^{-p^{2} \xi^{2}+\frac{q^{2}}{4 \xi^{2}}} \mathrm{~d} \xi=\frac{\sqrt{\pi}}{4 p}\left(\mathrm{e}^{-\mathrm{j} p q} \operatorname{erfc}\left(p r-\mathrm{j} \frac{q}{2 r}\right)\right. \\
&\left.+\mathrm{e}^{\mathrm{j} p q} \operatorname{erfc}\left(p r+\mathrm{j} \frac{q}{2 r}\right)\right)
\end{aligned}
$$

after some algebraic manipulations, (20b) becomes

$\Psi_{1}=\frac{1}{4 \Omega} \sum_{m, n} \mathrm{e}^{\mathrm{j} \mathbf{K}_{m n} \cdot \mathbf{R}} \sum_{ \pm} \frac{\mathrm{e}^{ \pm \gamma_{m n}\left(z-z^{\prime}\right)}}{\gamma_{m n}} \operatorname{erfc}\left(\frac{\gamma_{m n}}{2 E} \pm\left(z-z^{\prime}\right) E\right)$

where $\gamma_{m n}^{2}=K_{m n}^{2}-k^{2}$.

A direct application of (21) to (20c) with $p=R_{m n}>0$, $q=k$, and $r=E$ yields

$$
\Psi_{2}=\frac{1}{8 \pi} \sum_{m, n} \mathrm{e}^{-\mathrm{j} \mathbf{k} \cdot \boldsymbol{\rho}_{m n}} \sum_{ \pm} \frac{\mathrm{e}^{ \pm \mathrm{j} k R_{m n}}}{R_{m n}} \operatorname{erfc}\left(R_{m n} E \pm \frac{\mathrm{j} k}{2 E}\right) .
$$

In the above equations, $\sum_{ \pm}$designates the sum of the terms with + and - signs.

\section{OPTIMUM PARAMETER $E$ AND THE GF CONVERGENCE}

The complementary error function, which appears in both series $\Psi_{1}$ and $\Psi_{2}$, makes these series converge rapidly. The best choice for the splitting parameter $E$ is the one that balances the rate of decay of the two series, making $\Psi_{1}$ and $\Psi_{2}$ contribute an equal number of terms to the final value $\Psi$. The approximation 
to the optimal value of $E$ for 2-D orthogonal lattices is given by $E=\sqrt{\pi / \Omega}[13]$ and it can be shown that this expression remains valid for skewed lattices as well.

For high frequencies or, equivalently, large periodic spacings, the complementary error functions in the first terms $(m=n=$ $0)$ of both $\Psi_{1}$ and $\Psi_{2}$ will take large imaginary arguments and behave as $\exp \left(k^{2} /\left(4 E^{2}\right)\right)$. These two terms will have very large values that are approximately equal in amplitude, but of opposite signs and summing them up will lead to a severe loss of accuracy due to a finite machine precision. This problem can be remedied by requiring that $k^{2} /\left(4 E^{2}\right)$ be smaller than a maximum permitted exponent $H^{2}$. In that case, the value of the parameter $E$ has to be chosen as

$$
E=\max \left(\sqrt{\frac{\pi}{\Omega}}, \frac{k}{2 H}\right) .
$$

Consider a skewed lattice with $a_{1}=a_{2}$ and $\alpha=\angle\left(\mathbf{a}_{1}, \mathbf{a}_{2}\right)=$ $\pi / 3$. Let the source position be fixed at $\mathbf{r}^{\prime}=\left(\mathbf{a}_{1}+\mathbf{a}_{2}\right) / 2=$ $\mathbf{a}_{\Sigma} / 2$ and the observer be at the distance $R$ from the source along the diagonal $\mathbf{r}=\tau \mathbf{a}_{\Sigma}, \tau \in[0,1]$. Fig. 2 shows the number of significant digits of accuracy as the number of terms in both Ewald sums grows. The reference value for estimating the accuracy was computed for a large number of terms in both sums (729).

We can observe a very rapid convergence rate for small and $\lambda$-periodic spacings in both cases when the observer is close to the source $\left(R=0.01 a_{\Sigma}\right)$ and close to the edge of the unit cell $\left(R=0.4 a_{\Sigma}\right)$. Already for 25 terms, both sums achieve more than eight digits of accuracy as compared to the reference value. Fig. 3(a) illustrates the case of a large periodic spacing and the significant loss of accuracy that occurs due to a finite machine precision when a balanced optimal value of the parameter $E$ is used. However, when the parameter $E$ is based on the maximum exponent permitted, the accurate values of the GF are obtained [see Fig. 3(b)]. In that case, the sums are unbalanced and the modal sum needs much more terms to converge [see Fig. 3(c)].

The accurate numerical evaluation of the complex complementary error function can be done using very efficient numerical algorithms [30], [31]. A computationally efficient way of implementing GFs in a computer code is to pre-compute them at each frequency point and tabulate the values in a rectangular 3 -D grid along $x-x^{\prime}, y-y^{\prime}$, and $z-z^{\prime}$. The GF values needed to compute the numerical integrals in the MoM matrix are then retrieved from the table using interpolation routines (in our case, quadrature interpolation [31]). With a dense enough grid and nonuniform (cubic) distribution of points in the source region, the interpolation leads to very accurate results and much faster computation than if the GFs are evaluated directly.

\section{NUMERICAL RESULTS}

\section{A. Jerusalem Cross}

In the first example, we consider the problem of a freestanding planar array of perfectly conducting Jerusalem-cross shaped patches. Fig. 4 shows the magnitude and phase of the reflection coefficient for the Floquet TEM mode with the electric field oriented in the $x$-direction $\left(\mathrm{TEM}_{x}\right)$. The number
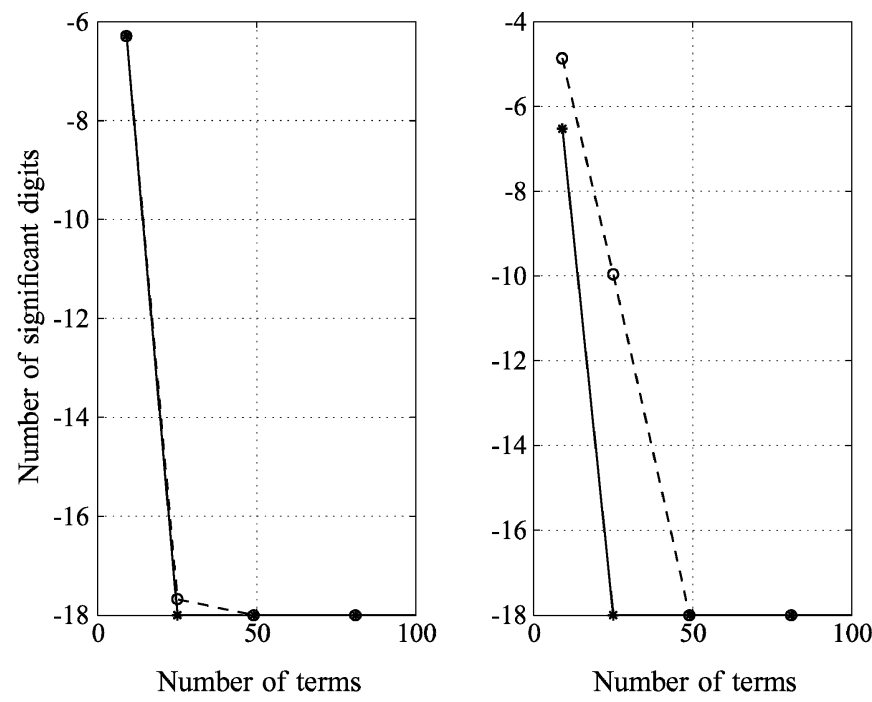

(a)
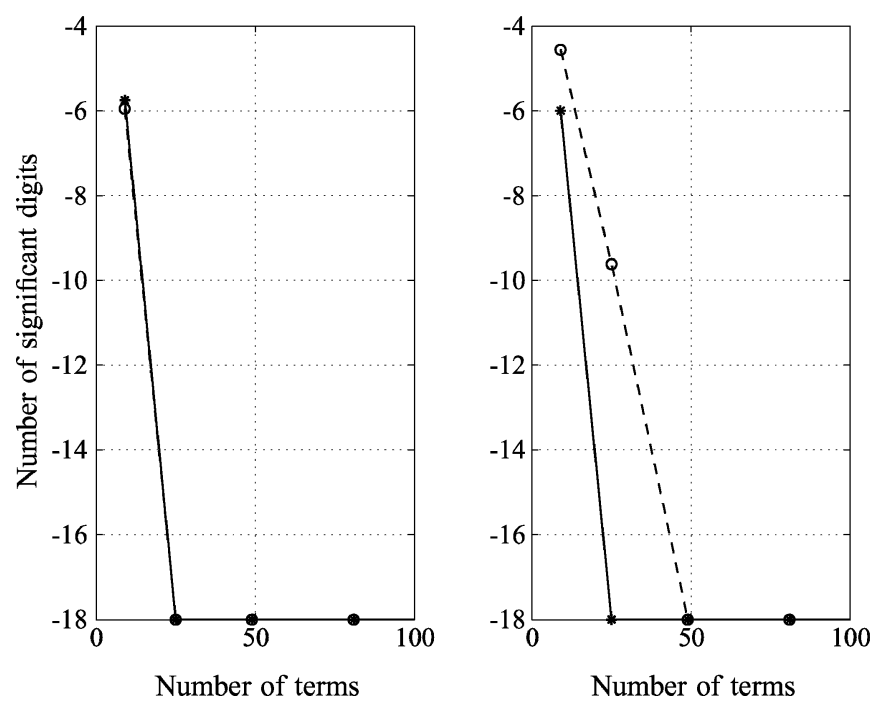

(b)

Fig. 2. Number of significant digits of accuracy as compared to the reference value computed using a very large number of terms. Both sums $\Psi_{1}$ (solid line *) and $\Psi_{2}$ (dashed line $\circ$ ) have very fast convergence and need approximately the same number of terms to achieve a given accuracy. (a) $a_{1}=a_{2}=\lambda / 5$ and (b) $a_{1}=a_{2}=0.99 \lambda$ for $E=E_{0} . R=0.01 a_{\Sigma}$ (left) and $R=0.4 a_{\Sigma}$ (right).

of terms in both Ewald sums was set to 25. Further increasing the number of terms did not show any changes in the reflection coefficient. The whole structure was discretized into 323 triangular cells to ensure accurate results in the entire frequency band. We compare the results obtained using our approach (solid line) with the results obtained using the spectral-domain approach [1] (dashed line) and the results obtained using the GSM [10] (circles). Good agreement with the reference results can be observed in both magnitude and phase of the reflection coefficient.

\section{B. Transmission Through Rectangular Apertures}

We calculate the transmission through a perfectly conducting screen perforated with rectangular apertures. The numerical example [26], used as a reference, has the following parameters: 


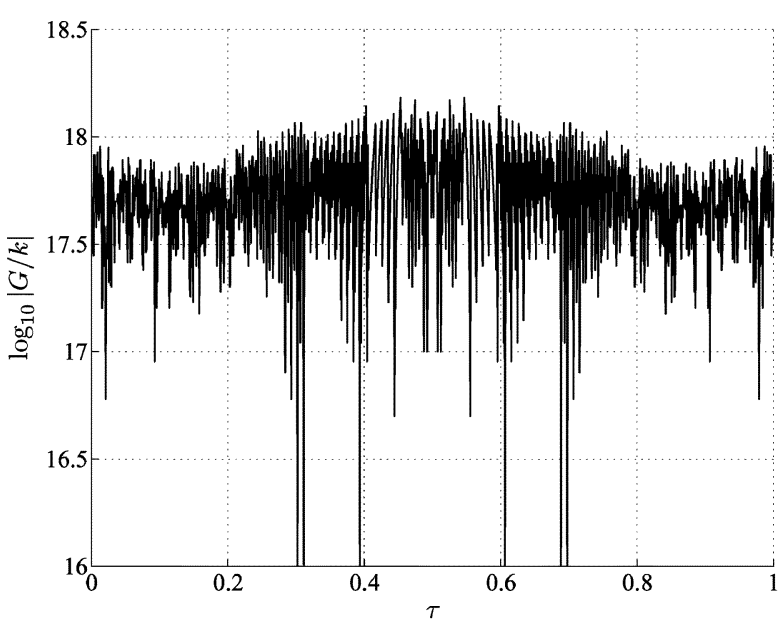

(a)

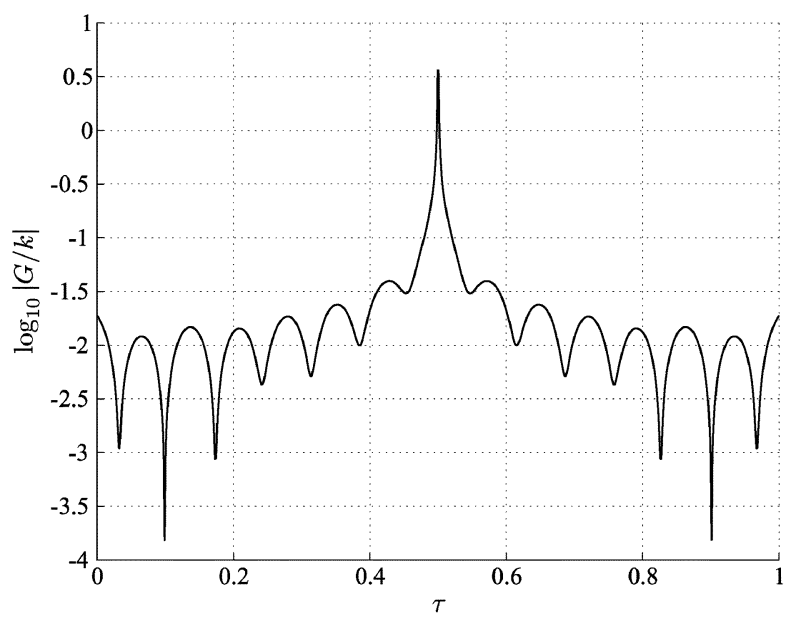

(b)
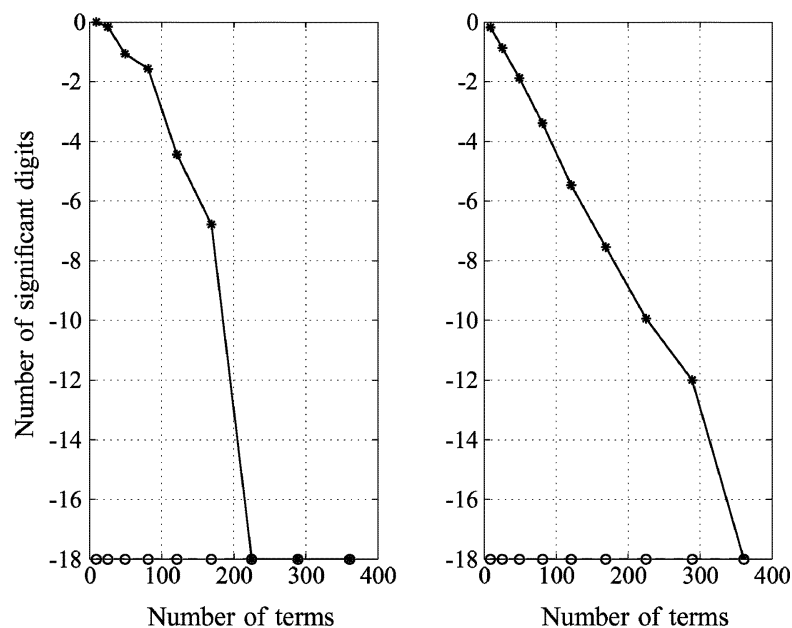

(c)

Fig. 3. (a) For large periodic spacing $a_{1}=a_{2}=5 \lambda$, significant loss of accuracy is observed if $E=E_{0}$ due to a finite machine precision. (b) When the parameter $E=2.75 E_{0}$ is based on the maximum exponent permitted $\left(H^{2}=9\right)$, accurate values of the GF are obtained. (c) In this case, the modal sum needs a larger number of terms to converge (solid lines with $*$ ). $R=0.01 a_{\Sigma}$ (left) and $R=0.4 a_{\Sigma}($ right $)$.

$a_{1}=20 \mathrm{~mm}, a_{2}=20 / \sqrt{3} \mathrm{~mm}, \alpha=30^{\circ}, l=12 \mathrm{~mm}$, $w=1.2 \mathrm{~mm}$, and the lattice vector $\mathbf{a}_{1}$ being in the $x$-direction.

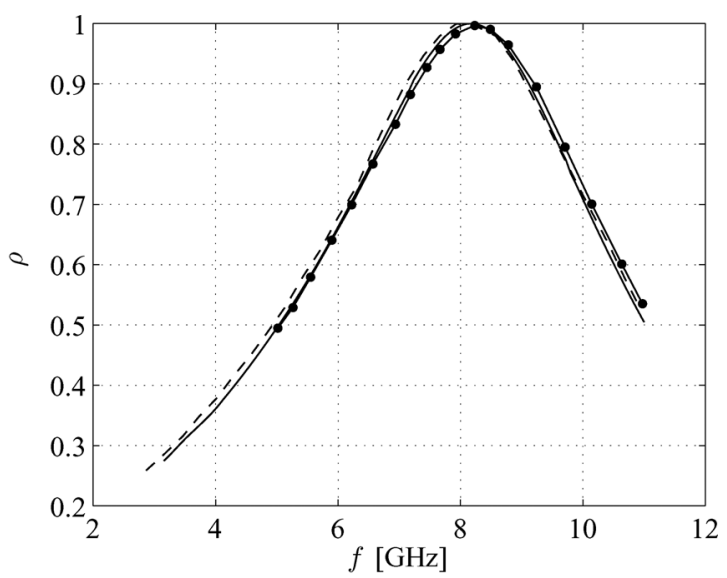

(a)

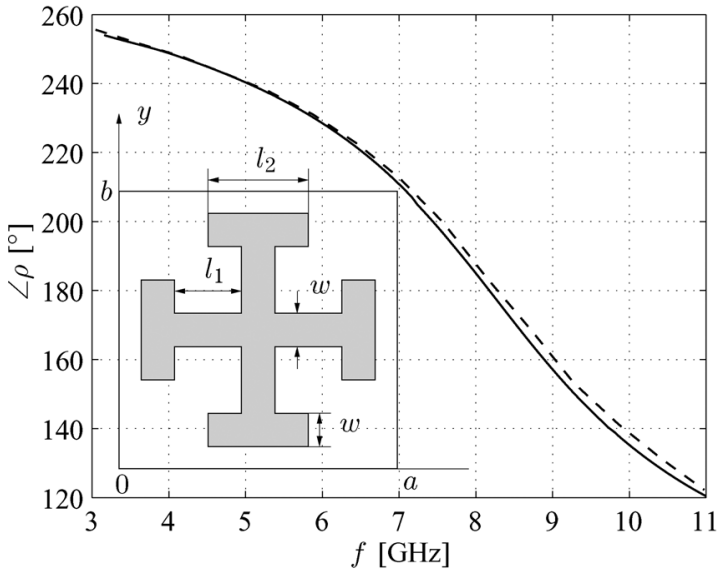

(b)

Fig. 4. (a) Magnitude and (b) phase of the reflection coefficient as a function of frequency for a normal incidence $\mathrm{TEM}_{x}$ illumination. Results obtained using the presented theory are denoted via solid lines, the values taken from [1] are denoted via dashed lines, and the values taken from [10] are denoted via circles. The inset shows a unit cell of the freestanding array of conducting patches in the shape of a Jerusalem cross. Dimensions are in millimeters: $w=1.9, l_{1}=3.8$, $l_{2}=5.7, a_{1}=a_{2}=15.2$.

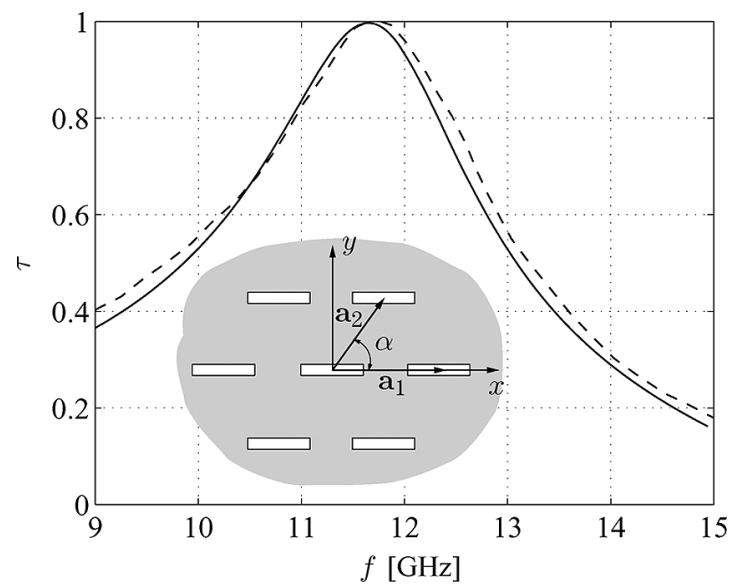

Fig. 5. Transmission coefficients of incident plane wave with the $E$-field perpendicular to the $x z$-plane and with the incident angle $\theta=60^{\circ}$. The results obtained using presented theory are denoted via solid lines and the values taken from [26] are denoted via dashed lines.

Fig. 5 shows the transmission coefficients of the plane wave that correspond to the $\mathrm{TE}_{00}$ Floquet mode with the incident angle 


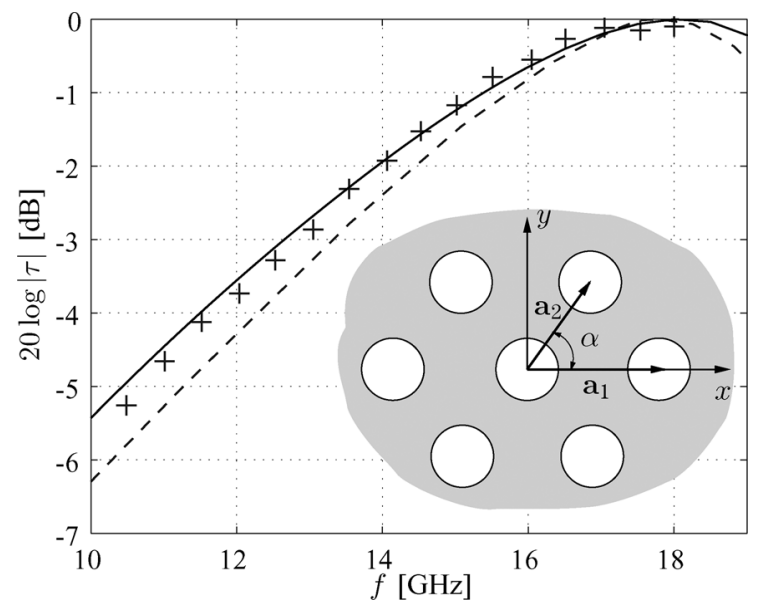

Fig. 6. Transmission coefficient of a normal incidence plane wave with $E$-field parallel to the $y$-axis. The results obtained using presented theory (solid lines) are compared to the simulations (dashed lines) and measured values $(+)$ taken from [32]. The inset shows the geometry layout of the 2-D array of circular holes in thin conducting screen. Diameter of the holes $D=12 \mathrm{~mm}, a_{1}=$ $a_{2}=10 \sqrt{3} \mathrm{~mm}$, and $\alpha=60^{\circ}$.

$\theta=60^{\circ}$. The results converged for 25 terms in both Ewald sums and 78 triangular cells used to discretize the geometry.

\section{Conducting Screen Perforated With Circular Holes}

The transmission of a plane wave incident on a thin perfectly conducting screen perforated periodically with circular holes is considered. The geometry of the problem is defined in the inset of Fig. 6. The reflection and transmission coefficients for a plane wave with the $E$-field parallel to the $y$-axis at normal incidence $\left(\mathrm{TEM}_{y}\right.$ mode) is plotted as a function of frequency in Fig. 6. A very good agreement with the measured values [32] can be noticed. Here, only nine terms in both Ewald sums give already convergent results for a mesh density with 178 triangular cells.

\section{Cross-Shaped Bandpass Filter}

Next, we analyze the bandpass filter formed by periodic cross-shaped holes with a resonance frequency of $280 \mathrm{GHz}$ [11]. The transmission coefficients for a plane wave with the $E$-field parallel to the $x$-axis at normal incidence $\left(\mathrm{TEM}_{x}\right.$ mode) is plotted as a function of frequency in Fig. 7. Solid lines represent the results obtained using our technique, dashed lines represent the results using the simulation technique of [11], and the measured values are denoted via pluses $(+)$. Very good agreement with the reference results can be observed. The number of terms in both Ewald sums was set to nine up to $f=687.5 \mathrm{GHz}$. Above this limit, the balanced value of the parameter $E=E_{0}$ results in loss of accuracy (Section VI). In order to avoid this, we use $E=2.75 E_{0}$ that corresponds to the maximum permitted exponent of $H^{2}=9$. Since the value of the splitting parameter is now unbalanced, we need more terms in the modal sum (49) than in the image one (9) to ensure the GF convergence. The thickness of the metallic foil has not been taken into account in our simulations.

\section{E. Photonic Crystal of Metallic Rods}

Now we consider a photonic crystal structure formed by a 2-D lattice of 3-D finite-length rods, as shown in the inset of

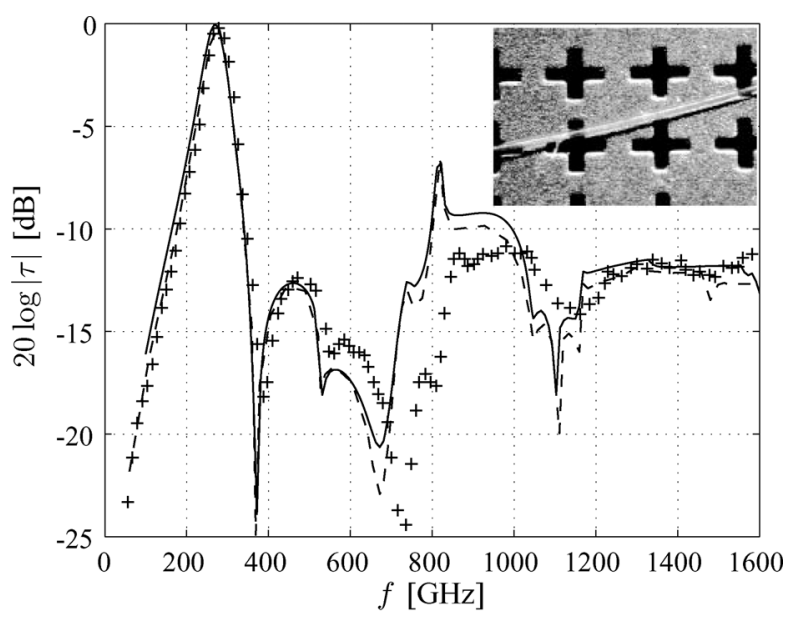

(a)

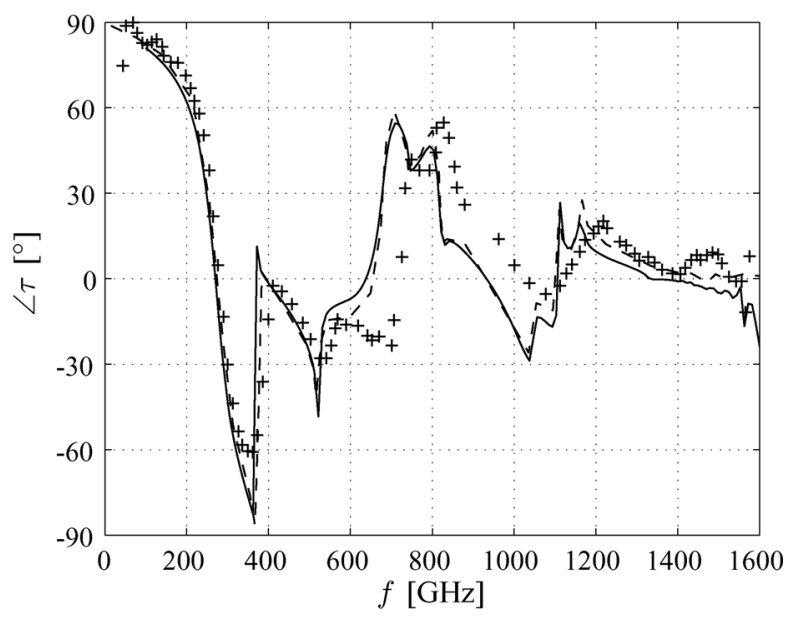

(b)

Fig. 7. (a) Magnitude and (b) phase of the filter's response: our technique (solid lines), numerical results from [11] (dashed lines), and the measured values from [11] $(+)$. The inset shows a photograph of a galvanized cross-shaped bandpass filter [11]. Square lattice period $a_{1}=a_{2}=810$, slot length $l=570$, slot width $w=160$, and foil thickness $t=10$. All dimensions given in micrometers.

Fig. 8. The structure is infinite in the $x$ - and $y$-directions and can have a finite number of lattice grids in the $z$-direction. The plane wave is propagating in the positive $z$-direction and has the electric field parallel to the rods. This excitation corresponds to the $\mathrm{TEM}_{x}$ Floquet mode. Fig. 8 presents the simulated transmission coefficient in the solid line versus the measured values taken from [33] and denoted by gray lines for the case of two lattice grids. GFs are tabulated in a rectangular $20 \times 10 \times 40$ grid and then evaluated using quadratic interpolation. The convergence of GFs is achieved already for nine terms in both Ewald sums. Increasing the number of terms in the Ewald sums only slightly increases the time needed to fill out the GF table. The mesh density fine enough to give the accurate results yields 528 unknowns. The time needed to solve this structure is $6.2 \mathrm{~s}$ per frequency point on a PC with Pentium IV 3.2-GHz processor, $1 \mathrm{~GB}$ of RAM, and Microsoft Windows $\mathrm{XP}$ operating system. It can be observed that the resulting transmission coefficient agrees very well with the experimental values. We have simulated the same problem using Ansoft's 


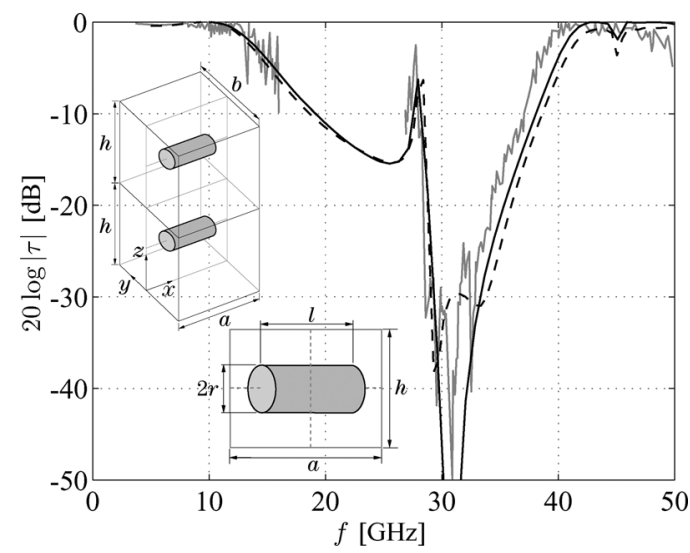

Fig. 8. Transmission coefficient for two layers of metallic photonic crystal grids. Spacing between the grids in the $z$-direction $h=6 \mathrm{~mm}$. Zero incident plane wave has the electric field parallel to the $x$-axis. The black solid line represents the simulated values. Gray solid lines represent measurements in 7-16and 27-50-GHz ranges taken from [33]. The dashed line is the result of simulations on HFSS. The inset shows the unit cell of the photonic crystal. The structure is periodic in the $x$ - and $y$-directions with rectangular lattice parameters $a_{1}=6.5$ and $a_{2}=6$. The radius of the rod is $r=0.75$ and its length $l=5$. All dimensions given in millimeters [33].
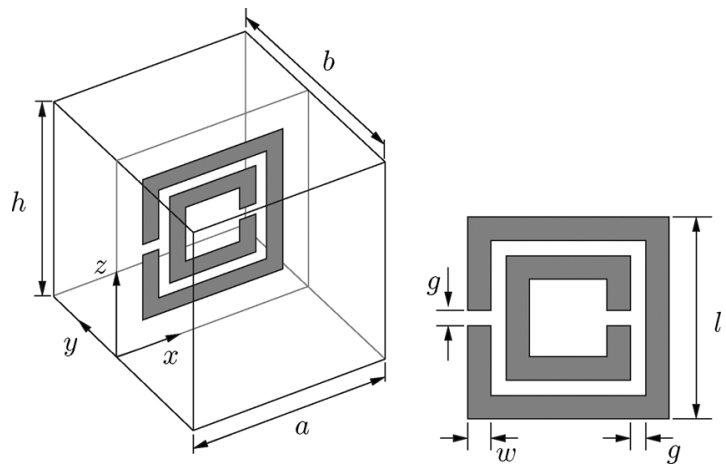

Fig. 9. Unit cell of periodic SRRs. The structure is infinite in the $x$ - and $y$-directions with periodicity $a_{1}=3.33$ and $a_{2}=3.66$, respectively. The height of a unit layer is $h=3.66$. The SRR consists of two split metallic rings with the following dimensions: $l=3, w=g=0.33$. All dimensions given in millimeters [34].

High Frequency Structure Simulator (HFSS) and the transmission coefficient denoted via dashed line in the same figure. The unit cell has ABCs on its top and bottom faces and they are placed far enough from the metallic rods (at $z= \pm 10 \mathrm{~mm}$ ) to ensure accurate results. The following simulation parameters were used: ten passes with a convergence of 0.02 per pass and the adaptive frequency $50 \mathrm{GHz}$, which leads to 6260 tetrahedra to achieve the convergence. The corresponding CPU time on the same PC is $26.1 \mathrm{~s}$ per frequency point, which means more than four times longer simulation as compared to that one of our approach, and no noticeable improvement in accuracy.

\section{F. Split-Ring Resonators (SRRs)}

A 2-D lattice of SRRs has been shown to give an effective permeability, which can be negative close to its resonance frequency. The transmission characteristics of arrays of SRRs have been extensively studied in [34] using the transfer-matrix method (TMM). Here, we perform the IE numerical analysis
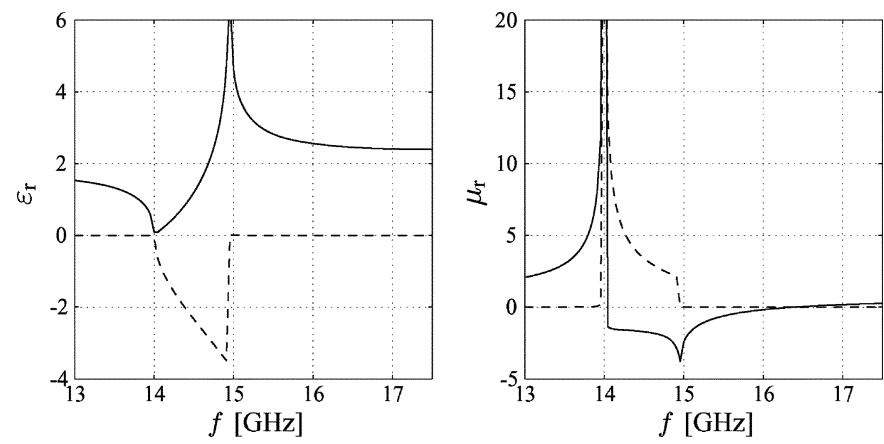

Fig. 10. Effective parameters for one layer of periodic SRRs. Solid lines denote real parts and dashed lines denote imaginary parts. EM wave propagates along the $z$-direction and its electric field is parallel to the $x$-axis. Note that $\mu_{\mathrm{r}}^{\prime}$ can be negative.

on such a periodic structure, whose geometry and dimensions are given in Fig. 9. The zero-incident EM plane wave has its electric field parallel to the $x$-axis. We have computed the reflection and transmission coefficients for the Floquet $\mathrm{TEM}_{x}$ mode again using only nine terms in the Ewald summations. Supposing that the layer of SRRs behaves as a homogeneous slab, we have then retrieved the effective permittivity and permeability of such a structure using the algorithm based on [35]. These results shown in Fig. 10 agree qualitatively very well with the results published in [34], and have a resonance interval in which the real part of the effective permeability $\mu=\mu^{\prime}-\mathrm{j} \mu^{\prime \prime}$ is negative.

\section{CONCLUSION}

We have presented in detail a space-domain IE analysis for 3-D metallic objects repeated along a 2-D lattice. The method uses Ewald's acceleration technique to speed up the convergence of the GFs. The values of GFs are pre-computed at each frequency point and tabulated in 3-D grids. The GF values are then retrieved from the table using 3-D interpolation routines, yielding very efficient numerical implementations with simulation times that, for this class of structures, outperform an FEM-based commercial software. A number of periodic structures with general skewed lattices under oblique and normal incidence plane-wave excitations were studied. The simulations show that, in most cases, only nine terms in both Ewald sums suffice to obtain results that agree very well with the measured or numerical results reported in the literature.

\section{APPENDIX A}

\section{Floquet Modes for General Skewed LatTice}

\section{A. Scalar-Mode Functions}

Let $\chi(\boldsymbol{\rho}), \boldsymbol{\rho}=x \hat{x}+y \hat{y}$, be a scalar-mode function that satisfies 2-D Helmholtz equation

$$
\left(\nabla_{\mathrm{t}}^{2}+K^{2}\right) \chi(\boldsymbol{\rho})=0
$$

with the following periodic BCs:

$$
\chi\left(\boldsymbol{\rho}+\boldsymbol{\rho}_{m n}\right)=\chi(\boldsymbol{\rho}) \mathrm{e}^{-\mathrm{jk} \cdot \boldsymbol{\rho}_{m n}} .
$$


TABLE I

VECTOR-MODE FUNCTIONS

\begin{tabular}{|c|c|}
\hline TE & TM \\
\hline \hline $\mathbf{e}_{t m n}=\frac{\mathrm{j}}{K_{m n}} \chi_{m n} \hat{z} \times \mathbf{K}_{m n}$ & $\mathbf{h}_{\mathrm{t} m n}=-\frac{\mathrm{j}}{K_{m n}} \chi_{m n} \hat{z} \times \mathbf{K}_{m n}$ \\
\hline $\mathbf{h}_{\mathrm{t} m n}=-\frac{\mathrm{j}}{K_{m n}} \chi_{m n} \mathbf{K}_{m n}$ & $\mathbf{e}_{\mathrm{t} m n}=-\frac{\mathrm{j}}{K_{m n}} \chi_{m n} \mathbf{K}_{m n}$ \\
\hline $\mathbf{h}_{z m n}=\frac{K_{m n}}{\gamma_{m n}} \chi_{m n} \hat{z}$ & $\mathbf{e}_{z m n}=\frac{K_{m n}}{\gamma_{m n}} \chi_{m n} \hat{z}$ \\
\hline
\end{tabular}

In the previous equations, $\nabla_{\mathbf{t}}^{2}=\left(\partial^{2} / \partial x^{2}\right)+\left(\partial^{2} / \partial y^{2}\right)$, $\mathbf{k}$ is the propagation vector, and $\boldsymbol{\rho}_{m n}=m \mathbf{a}_{1}+n \mathbf{a}_{2}$ is the translation vector of the skewed lattice defined by primitive vectors $\mathbf{a}_{1}$ and $\mathbf{a}_{2}$. The solution of this equation is found using the separation of variables and can be expressed as follows:

$$
\chi_{m n}=\frac{1}{\sqrt{\Omega}} \mathrm{e}^{\mathrm{j} \mathbf{K}_{m n} \cdot \boldsymbol{\rho}}
$$

where $\Omega=\left|\mathbf{a}_{1} \times \mathbf{a}_{2}\right|=a_{1} a_{2} \sin \alpha$ is the surface of the unit cell and $\mathbf{k}_{m n}=m \mathbf{k}_{1}+n \mathbf{k}_{2}$ is the translation vector of the reciprocal lattice.

\section{B. Vector-Mode Functions}

Vector-mode functions $\mathbf{e}_{m n}=\mathbf{e}_{t m n}+\mathbf{e}_{z m n}$ and $\mathbf{h}_{m n}=$ $\mathbf{h}_{\mathrm{tmn}}+\mathbf{h}_{z m n}$ for both TE and TM modes can be derived from the scalar-mode functions $\chi_{m n}$ using the formulas from [24] and the final expressions are compiled in Table I. In the case of zero incidence $\theta=0$, we have a TEM mode, whose electric vector is given by

$$
\mathbf{e}^{\mathrm{TEM}}=\frac{\mathrm{j}}{\sqrt{\Omega}}(\cos \phi \hat{x}+\sin \phi \hat{y}) .
$$

\section{REFERENCES}

[1] R. Mittra, C. H. Chan, and T. Cwik, "Techniques for analyzing frequency selective surfaces-A review," Proc. IEEE, vol. 76, no. 12, pp. 1593-1615, Dec. 1988.

[2] J. B. Pendry, A. J. Holden, D. J. Robbins, and W. J. Stewart, "Magnetism from conductors and enhanced nonlinear phenomena," IEEE Trans. Microw. Theory Tech., vol. 47, no. 11, pp. 2075-2084, Nov. 1999.

[3] D. R. Smith, W. J. Padilla, D. C. Vier, S. C. Nemat-Nasser, and S. Schultz, "Composite medium with simultaneously negative permeability and permittivity," Phys. Rev. Lett., vol. 84, no. 18, pp. 4184-4187, May 2000.

[4] A. Boag, Y. Leviatan, and A. Boag, "Analysis of diffraction from doubly periodic arrays of perfectly conducting bodies by using a patch-current model," J. Opt. Soc. Amer. A, vol. 7, no. 9, pp. 1712-1718, Sep. 1990.

[5] N.-W. Chen, B. Shanker, and E. Michielssen, "Integral-equation-based analysis of transient scattering from periodic perfectly conducting structures," Proc. Inst. Elect. Eng.-Microw. Antennas Propag., vol. 150, no. 2, pp. 120-124, Apr. 2003.

[6] I. Bardi, R. Remski, D. Perry, and Z. Cendes, "Plane wave scattering from frequency-selective surfaces by the finite-element method," IEEE Trans. Magn., vol. 47, no. 5, pp. 843-850, May 1999.

[7] S. D. Gedney, J. F. Lee, and R. Mittra, "A combined FEM/MoM approach to analyze the plane wave diffraction by arbitrary gratings," IEEE Trans. Microw. Theory Tech., vol. 40, no. 2, pp. 363-370, Feb. 1992.
[8] T. F. Eibert, J. L. Volakis, D. R. Wilton, and D. R. Jackson, "Hybrid FE/BI modeling of 3-D doubly periodic structures utilizing triangular prismatic elements and an MPIE formulation accelerated by the Ewald transformation," IEEE Trans. Antennas Propag., vol. 47, no. 5, pp. 843-850, May 1999.

[9] R. Pous and D. M. Pozar, "A frequency-selective surface using aperture coupled microstrip patches," IEEE Trans. Antennas Propag., vol. 39, no. 12, pp. 1763-1769, Dec. 1991.

[10] C. Wan and J. A. Encinar, "Efficient computation of generalized scattering matrix for analyzing multilayered periodic structures," IEEE Trans. Antennas Propag., vol. 43, no. 11, pp. 1233-1242, Nov. 1995.

[11] M. Bozzi, L. Perregrini, J. Weinzierl, and C. Winnewisser, "Efficient analysis of quasi-optical filters by a hybrid MoM/BI-RME method," IEEE Trans. Antennas Propag., vol. 49, no. 7, pp. 1054-1064, Jul. 2001.

[12] N. Kinayman and M. I. Aksun, "Comparative study of acceleration techniques for integrals and series in electromagnetic problems," Radio Sci., vol. 30, no. 6, pp. 1713-1722, Nov.-Dec. 1995.

[13] K. E. Jordan, G. R. Richter, and P. Sheng, "An efficient numerical evaluation of the Green's function for the Helmholtz operator on periodic structures," J. Comput. Phys., vol. 63, pp. 222-235, 1986.

[14] A. W. Mathis and A. F. Peterson, "Efficient electromagnetic analysis of a doubly infinite array of rectangular apertures," IEEE Trans. Microw. Theory Tech., vol. 46, no. 1, pp. 46-54, Jan. 1998.

[15] F. Capolino, D. R. Wilton, and W. A. Johnson, "Efficient computation of the 2-D Green's function for 1-D periodic structures using the Ewald method," IEEE Trans. Antennas Propag., vol. 53, no. 9, pp. 2977-2984, Sep. 2005.

[16] M.-J. Park and S. Nam, "Efficient calculation of the Green's function for multilayered planar periodic structures," IEEE Trans. Antennas Propag., vol. 46, no. 10, pp. 1582-1583, Oct. 1998.

[17] Y. Hua and T. K. Sarkar, "Generalized pencil-of-function method for extracting poles of an EM system from its transient response," IEEE Trans. Antennas Propag., vol. 37, no. 2, pp. 229-234, Feb. 1989.

[18] M. G. Silveirinha and C. A. Fernandes, "A new acceleration technique with exponential convergence rate to evaluate periodic Green's functions," IEEE Trans. Antennas Propag., vol. 53, no. 1, pp. 347-355, Jan. 2005.

[19] J. R. Mosig, "Arbitrarily shaped microstrip structures and their analysis with a mixed potential integral equation," IEEE Trans. Microw. Theory Tech., vol. 36, no. 2, pp. 314-323, Feb. 1988.

[20] _ _ "Integral-equation technique," in Numerical Techniques for Microwave and Millimeter-Wave Passive Structures, T. Itoh, Ed. New York: Wiley, 1989, ch. 3, pp. 133-213.

[21] S. M. Rao, D. R. Wilton, and A. W. Glisson, "Electromagnetic scattering by surfaces of arbitrary shape," IEEE Trans. Antennas Propag., vol. 30, no. 3, pp. 409-418, May 1982.

[22] D. R. Wilton, S. M. Rao, A. W. Glisson, D. H. Schaubert, O. M. Al-Bundak, and C. M. Butler, "Potential integrals for uniform and linear source distributions on polygonal and polyhedral domains," IEEE Trans. Antennas Propag., vol. AP-32, no. 3, pp. 276-281, Mar. 1984.

[23] R. D. Graglia, "On the numerical integration of the linear shape functions times the 3-D Green's function or its gradient on a plane triangle," IEEE Trans. Antennas Propag., vol. 41, no. 10, pp. 1448-1455, Oct. 1993.

[24] L. B. Felsen and N. Marcuvitz, Radiation and Scattering of Waves. Englewood Cliffs, NJ: Prentice-Hall, 1973.

[25] K. Kurokawa, "Power waves and the scattering matrix," IEEE Trans. Microw. Theory Tech., vol. MTT-10, no. 5, pp. 314-320, Sep. 1965.

[26] C.-C. Chen, "Transmission through a conducting screen perforated periodically with apertures," IEEE Trans. Microw. Theory Tech., vol. MTT-18, no. 9, pp. 627-632, Sep. 1970.

[27] I. Stevanović, P. Crespo-Valero, and J. R. Mosig, "An integral-equation technique for solving thick irises in rectangular waveguides," IEEE Trans. Microw. Theory Tech., vol. 54, no. 1, pp. 189-197, Jan. 2006.

[28] P. P. Ewald, "Die berechnung optischer und elektrostatischer gitterpotentiale," Ann. Phys., vol. 64, pp. 253-287, 1921.

[29] W. Gautschi, "Error function and Fresnel integrals," in Handbook of Mathematical Functions with Formulas, Graphs, and Mathematical Tables, M. Abramowitz and I. A. Stegun, Eds. New York: Dover, 1972, ch. 7, pp. 295-329.

[30] G. P. M. Poppe and C. M. J. Wijers, "More efficient computation of the complex error function," ACM Trans. Math. Softw., vol. 16, no. 1, pp. 38-46, Mar. 1990.

[31] IMSL Fortran Subroutines for Mathematical Applications. Visual Numerics Inc., Houston, TX, 1997. [Online]. Available: http://vni.com 
[32] C.-C. Chen, "Diffraction of electromagnetic waves by a conducting screen perforated periodically with circular holes," IEEE Trans. Microw. Theory Tech., vol. MTT-19, no. 5, pp. 475-481, May 1971.

[33] J.-M. Loutrioz, A. de Lustrac, F. Gadot, S. Rowson, A. Chelnokov, T. Brillat, A. Ammouche, J. Danglot, O. Vanbésian, and D. Lippens, "Toward controllable photonic crystals for centimeter- and millimeterwave devices," J. Lightw. Technol., vol. 17, no. 11, pp. 2025-2031, Nov. 1999.

[34] T. Koschny, P. Markoš, D. R. Smith, and C. M. Soukoulis, "Resonant and antiresonant frequency dependence of the effective parameters of metamaterials," Phys. Rev. E, Stat. Phys. Plasmas Fluids Relat. Interdiscip. Top., vol. 68, 2003, 065602.

[35] X. Chen, T. M. Grzegorczyk, B.-I. Wu, J. Pacheco, Jr., and J. A. Kong, "Robust method to retrieve the constitutive effective parameters of metamaterials," Phys. Rev. E, Stat. Phys. Plasmas Fluids Relat. Interdiscip. Top., vol. 70, 2004, 016608.

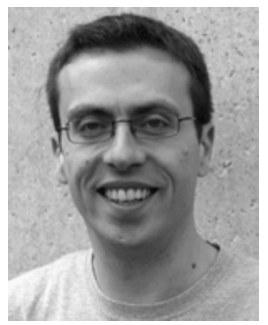

Ivica Stevanović (S'03-M'05) was born in Kruševac, Serbia, in 1976. He received the Dipl.Ing. degree in electrical engineering from the University of Belgrade, Belgrade, Serbia, in 2000, and the $\mathrm{Ph} . \mathrm{D}$. degree in electrical engineering from the Ecole Polytechnique Fédérale de Lausanne (EPFL), Lausanne, Switzerland, in 2005.

From 1998 to 2000, he was a Student Assistant with the Laboratory of Electronics, School of Electrical Engineering (ETF), University of Belgrade. In Summer 2000, he was a SURF Research Fellow with the Laser Interferometer Gravitational Wave Observatory (LIGO), California Institute of Technology (Caltech), Pasadena. In November 2000, he joined the Laboratory of Electromagnetics and Acoustics (LEMA), EPFL, where he is currently a Post-Doctoral Research Fellow. He is involved in several research projects for the European Space Agency (ESA), Swiss Federal Office of Communications (OFCOM), and the European Networks of Excellence on metamaterials (Metamorphose) and antennas (ACE).

Dr. Stevanović was the recipient of a scholarship presented by the Serbian Ministry of Education (1997-2000) and a research fellowship (NSF-REU-SURF) presented by Caltech.

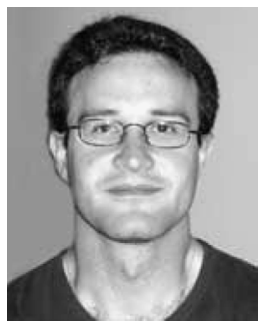

Pedro Crespo-Valero was born in Antas (Almería), Spain, in 1976. He received the Ingeniero de Telecomunicación degree from the Universidad Politécnica de Madrid (UPM), Madrid, Spain, in 2001, and is currently working toward the Ph.D. degree at the Ecole Polytechnique Fédérale de Lausanne (EPFL), Lausanne, Switzerland.

In February 2002, he joined the Laboratory of Electromagnetics and Acoustics (LEMA), EPFL, where he is a Research and Teaching Assistant. He is involved in several projects with the European Space Agency (ESA) and other European universities and industries. His research interests include the computer-aided design (CAD) of microwave passive devices and circuits.

Mr. Crespo-Valero is a member of Colegio Mayor Diego de Covarrubias, Universidad Complutense de Madrid (UCM), Madrid, Spain.

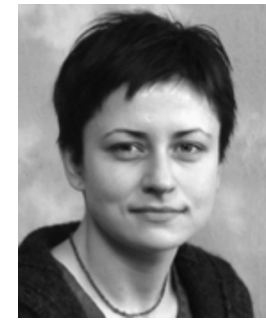

Katarina Blagović was born in Sarajevo, Bosnia and Herzegovina, in 1976. She received the Dipl.Ing. degree in electrical engineering from the University of Zagreb, Zagreb, Croatia, in 2001, and is currently working toward the Ph.D. degree at the Ecole Polytechnique Fédérale de Lausanne (EPFL), Lausanne, Switzerland.

In February 2002, she joined the Laboratory of Electromagnetics and Acoustics (LEMA), EPFL, where she is a Research and Teaching Assistant. She is involved in projects supported by the Swiss $\mathrm{Na}$ tional Science Foundation (SNF). Her research interests include computational electromagnetics and frequency selective surfaces.

Ms. Blagović was the recipient of a scholarship presented by the Croatian Ministry of Science, Education, and Sports (1996-2000) and a scholarship presented by the University of Zagreb (2000-2001).

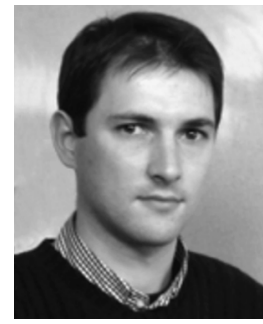

Frédéric Bongard was born in Yverdon, Switzerland, in 1978. He received the B.S. and M.S. degrees in electrical engineering from the Ecole Polytechnique Fédérale de Lausanne (EPFL), Lausanne, Switzerland, in 2003, and is currently working toward the Ph.D. degree at EPFL.

From 2003 to 2004, he was an Assistant with the acoustics portion of the Laboratory of Electromagnetics and Acoustics (LEMA), EPFL. Since October 2004, he has been with the electromagnetics part of LEMA, where he is currently a Research and Teaching Assistant. He is involved in projects for the European Space Agency (ESA) and the European Network of Excellence on metamaterials (Metamorphose). His research interests include the characterization of metamaterials and investigation of potential applications of metamaterials to the antenna field.

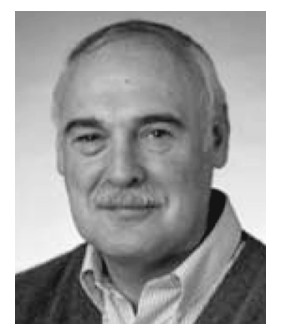

Juan R. Mosig (S'76-M'87-SM'94-F'99) was born in Cadiz, Spain. He received the Electrical Engineer degree from the Universidad Politecnica de Madrid, Madrid, Spain, in 1973, and the Ph.D. degree from the Ecole Polytechnique Fédérale de Lausanne (EPFL), Lausanne, Switzerland, in 1983.

In 1976, he joined the Laboratory of Electromagnetics and Acoustics, EPFL. Since 1991, he has been a Professor with EPFL, and since 2000, he has been the Head of the Laboratory of Electromagnetics and Acoustics (LEMA). In 1984, he was a Visiting Research Associate with the Rochester Institute of Technology, Rochester, NY, and Syracuse University, Syracuse, NY. He has also held scientific appointments with the University of Rennes, Rennes, France, the University of Nice, Nice, France, the Technical University of Denmark, Lyngby, Denmark, and the University of Colorado at Boulder. He is co-organizer and lecturer of yearly short courses in numerical electromagnetics in both Europe and the U.S. He is also responsible for several research projects of the European Space Agency (ESA). $\mathrm{He}$ has authored four chapters in books on microstrip antennas and circuits and over 100 reviewed papers. His research interests include EM theory, numerical methods, and planar antennas.

Dr. Mosig is a member of the Swiss Federal Commission for Space Applications and a member of the Executive Board of the European Network of Excellence ACE (2004-2007). He is the chairman of the European COST Project on Antennas (2003-2006). 